\title{
Identités et communautés de pratiques des chatteurs malgachophones dans les cybercafés de Tananarive (Madagascar)
}

Identities and Community of Practices of the Malagasy Users of Mediated Interactions in the Cybercafés of Antananarivo (Madagascar)

\section{Maud Verdier}

\section{OpenEdition \\ Journals}

Édition électronique

URL : http://journals.openedition.org/etudesafricaines/17796

DOI : 10.4000/etudesafricaines. 17796

ISSN : $1777-5353$

Éditeur

Éditions de l'EHESS

Édition imprimée

Date de publication : 3 octobre 2014

Pagination : 685-713

ISSN : 0008-0055

Référence électronique

Maud Verdier, "Identités et communautés de pratiques des chatteurs malgachophones dans les cybercafés de Tananarive (Madagascar) », Cahiers d'études africaines [En ligne], 215 | 2014, mis en ligne le 02 octobre 2016, consulté le 21 avril 2019. URL : http://journals.openedition.org/ etudesafricaines/17796 ; DOI : 10.4000/etudesafricaines.17796

Ce document a été généré automatiquement le 21 avril 2019

(c) Cahiers d'Études africaines 


\title{
Identités et communautés de pratiques des chatteurs malgachophones dans les cybercafés de Tananarive (Madagascar)
}

\author{
Identities and Community of Practices of the Malagasy Users of Mediated \\ Interactions in the Cybercafés of Antananarivo (Madagascar)
}

Maud Verdier

1 À partir de l'étude anthropologique des pratiques de chat dans les cybercafés de Tananarive, on remet en cause l'idée largement répandue que la médiatisation des interactions entraîne obligatoirement la création de nouvelles identités sociales évoluant dans des communautés virtuelles. Cette idée a comme origine le postulat d'une dichotomie off line/on-line permettant d'opposer la communauté virtuelle à la communauté réelle. Un point de vue anthropologique incite à refuser une approche théorique centrée sur une telle opposition réel/virtuel. Pour comprendre l'importance accordée par les participants à l'inscription dans un ici et maintenant, nous nous appuyons sur l'ethnographie approfondie des interactions médiatisées. Inscrite dans le champ de l'anthropologie linguistique (Hanks 1996), notre approche examine les pratiques des jeunes chatteurs malgachophones de Tananarive et s'appuie sur un corpus d'interactions chat filmées au sein des cybercafés de la capitale malgache, transcrites et traduites selon les conventions de l'analyse de conversation.

2 En nous appuyant sur l'observation des pratiques, nous étudions dans un premier temps la manière dont les identités entre jeunes chatteurs se construisent tout à la fois dans les chats et dans un environnement géographiquement et socialement situé. Dans un deuxième temps, nous montrons que les interactions médiatisées par ordinateur s'inscrivent aussi dans un espace social que l'on peut caractériser anthropologiquement, ce qui a des effets non négligeables sur les échanges; inversement, les chatteurs 
s'appuient pour interagir sur un certain nombre de présuppositions liées à leurs pratiques de chat.

\section{Les travaux sur les identités à l'œuvre dans les pratiques interactionnelles médiatisées}

Lorsque les chats commencent à devenir un objet de recherche à la fin des années 1980 (Baron 1984; Murray 1988; Ferrara et al. 1991; Herring et al. 1992), les chercheurs supposent que les communications de type chat, et plus généralement les nouvelles technologies, président à des changements sociaux (Kiesler \& Sproull 1987). La question de la communauté, associée à celle de l'identité, devient centrale (Byrne 1994; Baym 1995 ; Beaudouin \& Velkovska 1999; Werry \& Mowbray 2001; Baron 2004). Le thème de l'identité restant centré sur la dichotomie monde réel/monde virtuel (Zagalo et al. 2012), on étudie l'impact de ce dernier sur les constructions identitaires personnelles (Turkle 1995). Parce que les nouvelles technologies semblent introduire une subversion des frontières, on insiste sur l'enjeu social et politique : "Much of the recent scholarship is focused on the promise of new communications technologies to subvert social boundaries such as race, gender and ethnicity through text-based media that with hold certain identity markers » (Cook $2004: 104)$.

Un argument généralement avancé pour inciter les anthropologues à investir ce domaine de recherche est que les nouvelles technologies sont des produits culturels ayant permis l'émergence de nouvelles formes de communautés et de pratiques de communication (Escobar 1994; Wilson \& Peterson 2002). Mais la promesse des transformations est remise en cause. Malgré les prédictions de certains chercheurs (Crystal 2004), les bouleversements ont été assez relatifs et on constate que ces pratiques s'inscrivent en définitive dans des pratiques déjà existantes (Wilson \& Peterson 2002: 462). Dans le domaine de l'anthropologie linguistique, quelques études portent sur les nouvelles technologies, en particulier sur la relation entre l'utilisation d'une (ou des) langue(s), leur statut et les idéologies linguistiques en rapport avec les nouvelles technologies (Cook 2004 ; McIntosh 2010 ; Verdier 2013). Du fait que celles-ci constituent une révolution, à l'instar de celle provoquée par l'introduction de la presse écrite, on se donne alors pour objet d'étude la compréhension des liens entre nouvelles technologies et évolution des conventions sociales, avec les modifications de la nature des interactions sociales (Keating \& Mirus 2003) et les changements linguistiques que ces pratiques de communication induisent (Jones \& Schieffelin 2009).

Malgré tout, les pratiques liées aux nouvelles technologies invitent l'anthropologue à reprendre certains concepts dont celui d'identité. Il ne s'agit pas ici de déconstruire cette catégorie comme le postulent certains chercheurs de la «cyberculture " (Haraway 1991; Escobar 1994). C'est par exemple la position de M. Poster (1990:8) pour qui les nouvelles technologies obligent les anthropologues à remettre en cause leurs catégories étant donné que la «technoscience» modifie les frontières posées habituellement entre le naturel, l'organique, le technique et le textuel (ou encore la culture). Parce que le cyberespace est réifié, "pensé comme univers parallèle et distinct du "réel" » (Pastinelli 2011: 35), il est fréquent de trouver dans les travaux l'idée que la combinaison de l'anonymat et la désincarnation des corps créent un environnement dans lequel un nouveau mode de production identitaire peut émerger (Bargh et al. 2002; McKenna et al. 
2002 ; Zhao et al. 2008). On en vient alors à privilégier le caractère de jeu identitaire des personnes utilisant les nouveaux médias de communication et, de manière générale, on considère que les sites de rencontre sur l'Internet sont des lieux « anonymes " où l'on peut cacher ou au contraire dévoiler sa personnalité, et où, quoi qu'il arrive, on laisse des traces. De là émerge la notion d'« identité numérique » comme collection de celles-ci (Georges 2011), nécessairement pensée comme plurielle. Ce faisant, on ne fait que thématiser le sens commun qui pose une différence fondamentale entre les identités en situation de face à face et celles en situation de rencontre virtuelle, en raison de la difficulté à mobiliser (ou de la facilité à manipuler) des indices de contextualisation sociaux et l'hexis corporelle des interactants.

Plutôt que de considérer que les identités sont jouées, données, négociées voire contestées, nous postulons qu'elles sont d'abord située ${ }^{1}$ : lorsqu'ils interagissent, les participants sont toujours ancrés dans un espace social et une temporalité particulière. Notre étude s'inscrit dans les travaux qui, loin de montrer que la médiatisation des interactions entraînerait obligatoirement une modification des relations sociales et une restructuration des sujets qui les constituent, dotés de nouvelles identités virtuelles, soulignent au contraire que les rôles sociaux sont toujours à l'œuvre dans les communautés on-line (Correll 1995; Wheeler 2001). Il s'agit principalement des recherches qui explorent la manière dont les interactions on-line sont influencées par les off-line en termes de genre notamment. A. Markham (1998) a ainsi montré la manière dont les identités on-line sont directement liées à la notion de crédibilité. Les chercheurs n'ont cependant pas poussé jusqu'au bout la déconstruction de la dichotomie off line/on-line (Wilson \& Peterson 2002 : 456). On propose une approche anthropologique permettant de s'extraire de l'opposition réel/ virtuel.

\section{L'enquête ethnographique dans les cybercafés de Tananarive}

7 Certains chercheurs postulent la nécessité de nouvelles formes d'enquête et l'élaboration de nouveaux concepts pour l'observation des pratiques liées aux nouvelles technologies (Jones 1998; Axel 2006). A. Markham (2013: 435) considère qu'il faut modifier en profondeur les techniques ethnographiques traditionnelles (comprendre, celles élaborées par B. Malinowski), le problème étant celui de la définition même du terrain d'enquête pour qui s'intéresse aux identités et aux formations culturelles: "When identities and cultural formations are located in or made of information flows through global networks, where do we demarcate the boundaries of "the field" ? " La remise en cause de la notion de terrain s'accompagne d'un questionnement sur les méthodes d'observation, les carnets de terrain, la manière dont peuvent être menés des entretiens et la nature même des corpus. Les questionnements concernant les spécificités de l'enquête de terrain prenant pour objet des usages des communications électroniques (Pastinelli 2011) peuvent mener à proposer une nouvelle ethnographie baptisée netnographie (Kozinets 2002).

8 Ces propositions méthodologiques participent d'une conception des pratiques médiatisées reproduisant la dichotomie off line/on-line avec pour effet d'ignorer le contexte sociologique des acteurs dont on étudie les pratiques. Les études sur les interactions médiatisées, qui privilégient généralement la trace écrite des échanges et des entretiens, parce qu'elles ne se donnent pas un accès au déroulement en temps réel de 
l'activité, impliquent le plus souvent des interactions décontextualisées. Or une étude adéquate des situations de chat suppose de prendre en compte l'activité en tant que telle, dans son déroulement temporel, au sein d'un environnement particulier qui dépasse le cadre de l'écran d'ordinateur et s'inscrit plus largement dans un lieu public comme le cybercafé. Nous avons donc opté pour une approche ethnographique qui s'appuie sur les acquis de l'anthropologie linguistique et qui inclut la prise en compte du contexte socioculturel des pratiques d'interactions médiatisées par ordinateur ${ }^{2}$. Notre méthodologie suppose de partir de données «naturelles » enregistrées par vidéo pour capter toutes les dimensions de la multimodalité de l'interaction.

À Tananarive, nous avons rencontré les jeunes, adolescents et jeunes adultes qui chattent dans les cybercafés, n'ayant pas de connexion à domicile. Dans ces lieux, les personnes chattent, consultent leur courrier, vérifient leurs SMS, écoutent de la musique, regardent des sites et discutent avec leurs amis présents dans le lieu : l'activité de chat s'inscrit par conséquent, au sein du cybercafé, dans un ensemble d'activités diverses. Les usagers de l'Internet que nous avons rencontrés habitent un monde globalisé où la modernité va de pair avec l'utilisation des nouvelles technologies et une connexion permanente. Soulignons à cet égard que l'usage des chats est lié à l'utilisation des communications par SMS (à l'époque de l'enquête, les ventes de téléphones portables sont en croissance constante): un usager de chat peut envoyer un sms à la personne avec qui il est en relation, ou bien lui envoyer un SMS via un site Internet qui permet d'écrire et d'envoyer des messages de ce type. Les pratiques de l'Internet dans les cybercafés de Tananarive, loin d'être isolées, s'inscrivent au contraire dans un vaste ensemble d'activités propres à leur classe d'âge (qui varie de 15 à 22 ans environ) : regarder l'émission de télé-réalité Pazzapa, diffusée sur la chaîne RTA; aller se promener à la campagne; écouter de la musique; aller voir des ami(e)s; aller à la messe; et aussi faire la cuisine; faire le marché ; s'occuper de ses frères ou sœurs ou de ses grands-parents; faire son travail scolaire, etc. Dans cet ensemble disparate d'activités et de réseaux sociaux qui y sont liés, l'usage du chat est pour ces jeunes adultes un moyen de se connecter facilement avec le monde entier, ce qui explique l'engouement pour les sites de rencontres. Toutefois, l'Internet n'est pas seulement un lieu de rencontres lointaines, fugaces, car elles donnent lieu parfois, du fait de connections répétées avec les mêmes personnes, à un certain type de lien social. En outre, cette pratique est aussi l'occasion de rencontres locales avec des personnes situées dans la même ville, voire dans le même cybercafé. Nombre de chatteurs que nous avons rencontrés surfent sur rta.org, le site de la station de télévision alors très en vogue au moment de notre enquête chez les jeunes Tananariviens, un site principalement malgachophone avec des jeunes résidant pour la plupart à Madagascar. Dans ce cas, il est possible que des numéros de téléphone s'échangent, voire qu'un rendez-vous soit pris.

Dans cet article, nous présentons des conversations privées sur les sites de messageries $\mathrm{m}$ IRC $^{3}$ (rta.org ou wanadoo.mg) $)^{4}$. Selon qu'elles concernent un chat avec une personne située dans un pays étranger ou qui habite Tananarive, la finalité des interactions n'est pas tout à fait la même. Principalement une occasion de discussions amicales dans le premier cas, l'enjeu dans le second cas ne peut plus en rester à une relation virtuelle mais vise souvent à créer les conditions d'un contact futur en face à face : il s'agit d'obtenir des numéros de téléphone et éventuellement un rendez-vous.

11 La plupart des jeunes privilégient le chat en " $\mathrm{pv}$ » (privé) et n'interviennent que très rarement dans la room (salon public). S'ils le font, c'est pendant un temps restreint par 
rapport à la durée de leur séance de chat et souvent parce que personne ne leur a répondu en privé. Lorsqu'ils ont trouvé quelqu'un sur le " pv», ceux qui ont un logiciel de type Messenger peuvent proposer de passer sur Yahoo ou MSN Messenger. Concernant spécifiquement les interactions chat en privé " pv ", l'internaute entre dans un site qui lui permet d'accéder à un salon (room) à partir duquel il entre en contact avec d'autres internautes, soit en interagissant dans le salon, soit en ouvrant des fenêtres applet qui lui permettent de dialoguer en privé. Le dispositif interactionnel constitué par le site de chat offre ainsi un espace d'interactions diffuses et non focalisées (room) et permet en même temps l'émergence d'interactions focalisées de type duel « $p v »^{5}$ (de Fornel 1989). Pour ce faire, l'internaute se connecte sur le site de son choix et pénètre d'abord dans un espace où plusieurs autres internautes sont déjà en interaction (soit dans la room - ce qui est visible par tous - soit en «pv» - ce qui est invisible). Puis il/elle choisit parmi une liste de personnes connectées celui/celle avec qui il/elle veut rentrer en contact. Dès que l'internaute clique sur un pseudo, ou bien si un autre pseudo lui envoie un message, le nouvel entrant se trouve alors immédiatement dans un espace d'interaction focalisée et au sein d'un espace interactionnel plus large. C'est ce que montrent les photographies qui suivent, extraites de notre corpus :

ILL. 1. <OCEANNEMAYA> CHATTE SUR RAT.ORG EN PRIVÉ. EN HAUT, <OCEANNEMAYA> VUE DE FACE, EN BAS, LA FENÊTRE D'APPLET DU «PV »
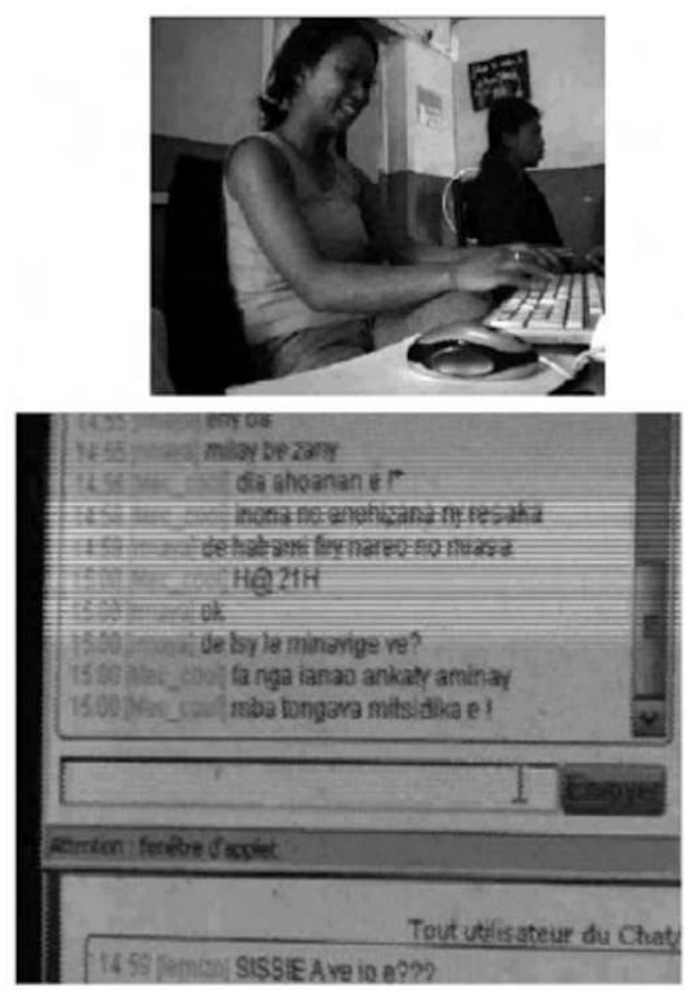
ILL. 2. <FLEURDEMIEL> CHATTE SUR WANADOO.MG (5 CONVERSATIONS EN PRIVÉ SONT OUVERTES SIMULTANÉMENT)

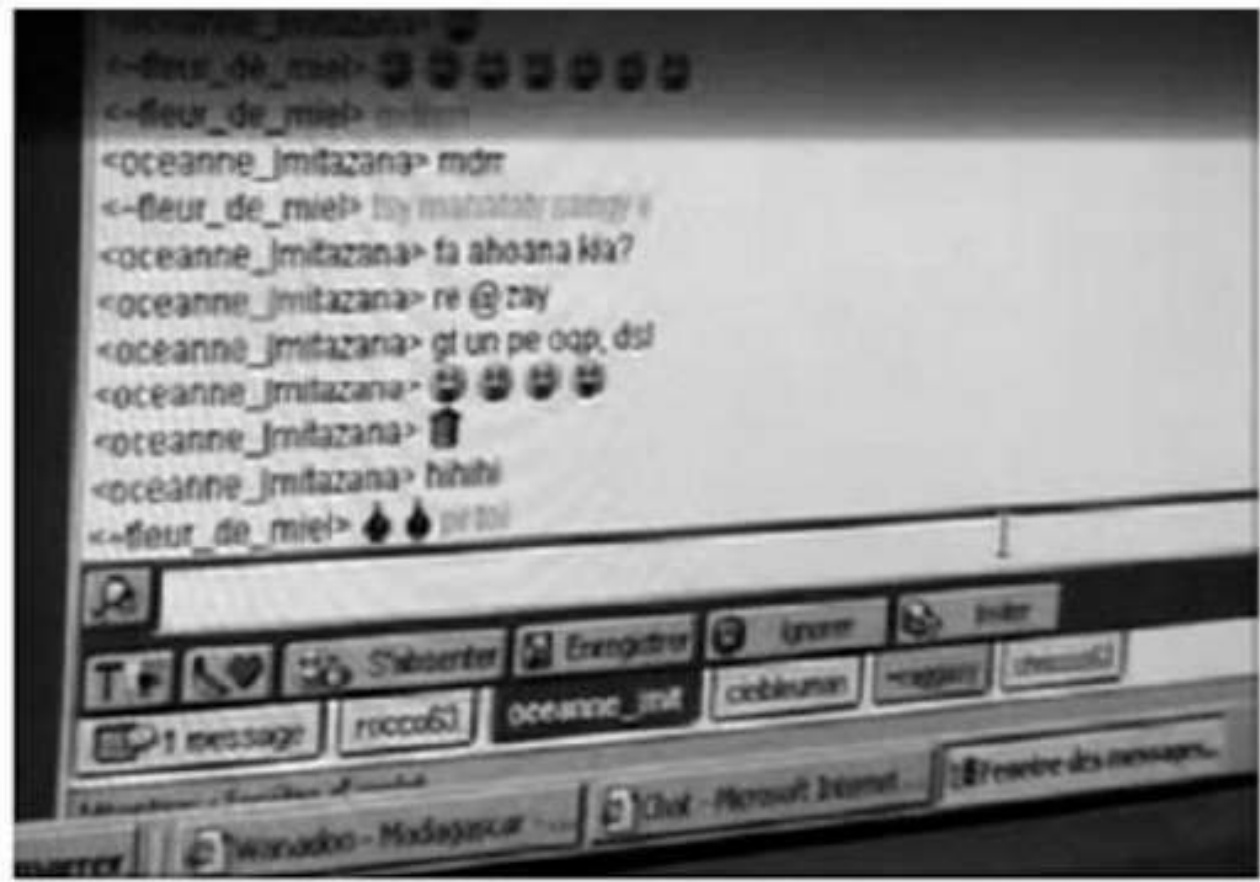

12 Au bout d'un temps plus ou moins long selon les internautes, plusieurs conversations privées sont ouvertes sur l'écran ainsi que l'illustre la photographie suivante, où la chatteuse <maya $>$ a ouvert trois conversations en privé sur rta.org. 


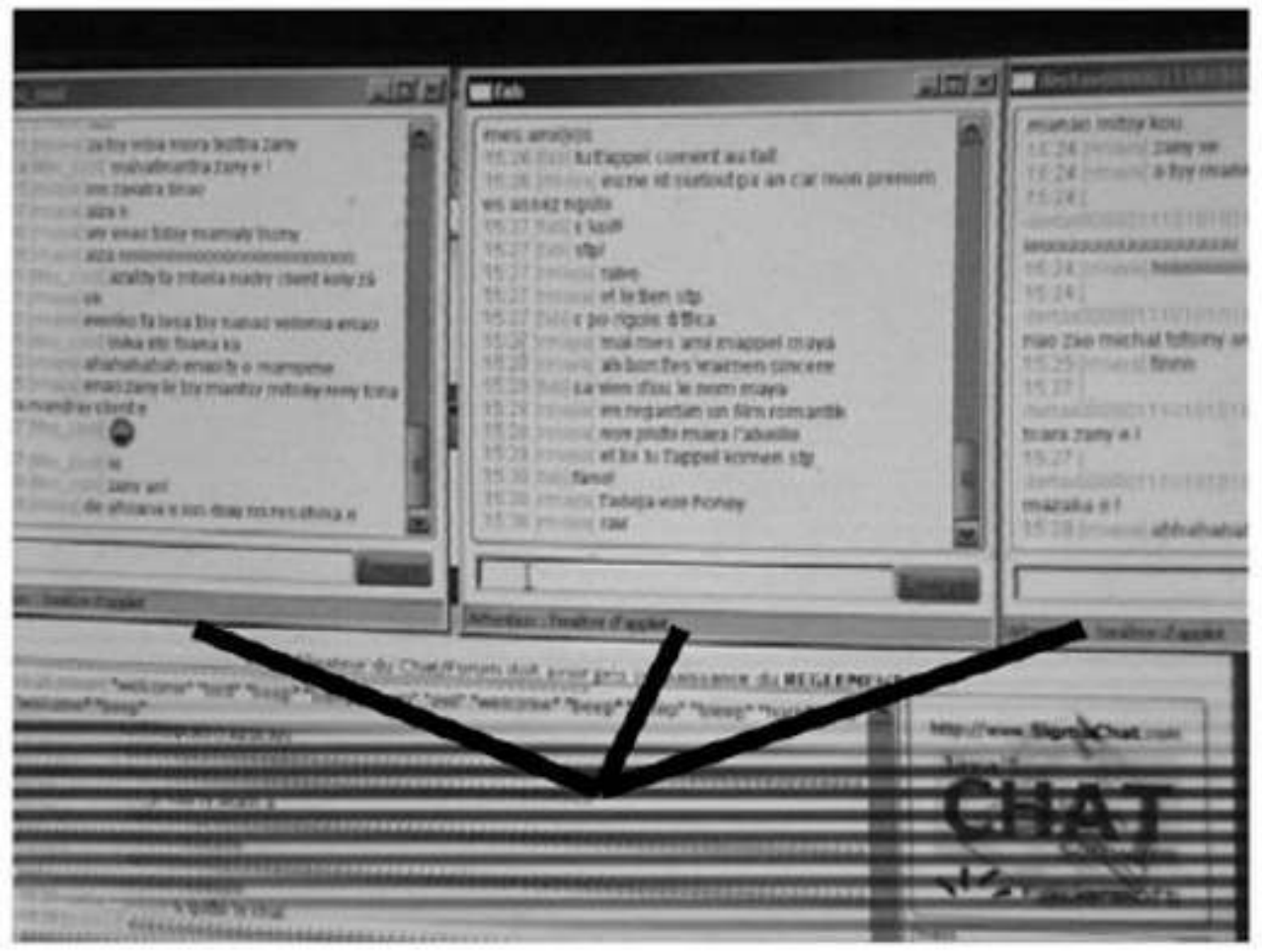

<Maya> a ouvert trois conversations en privé (chacune dans une fenêtre applet) en se connectant sur le site de rta.org

Dans un tel environnement interactionnel, de quelle manière les identités entre jeunes chatteurs se construisent-elles? C'est ce que nous étudions dans ce qui suit.

\section{Identités émergentes et identités situées}

14 L'identité mise en œuvre par les usagers de l'Internet n'est pas nationale, encore moins ethnique. Toutefois, elle n'est pas non plus réinventée à chaque connexion et à chaque interaction avec un autre chatteur. Bien qu'appréhendée au premier abord par le pseudonyme qui joue un rôle non négligeable dans la mise en scène de soi, l'identité de chacun se construit au fur et à mesure de l'interaction tout en étant située dans un espace et un temps particuliers. Certains phénomènes interactionnels n'ont de sens que si l'on conçoit les usagers des chats comme des personnes ancrées dans des contextes locaux, à chaque fois particularisés : c'est pourquoi la localisation réciproque des interactants peut jouer un rôle central dans la construction d'une relation sociale qui prend progressivement appui sur l'espace physique des interactants. Même dans le cas d'une relation d'interconnaissance entre familiers ou d'une situation institutionnelle mobilisant des rôles sociaux, les identités ne sont cependant pas fixes ou monolithiques: elles s'incarnent dans des pratiques qui se modifient selon les caractéristiques de la situation même dans laquelle les participants sont engagés. 


\section{Les pseudonymes}

15 À cet égard, le pseudonyme participe de la construction des identités. Dans ces situations, les interlocuteurs qui ne se connaissent pas, ou peu, construisent peu à peu au cours de cette rencontre et de manière réciproque une identité. Le pseudonyme est le premier indice de cette coprésence immédiate. À ce titre, il occupe un statut identique à celui du temps qu'il fait, ressource toujours mobilisable dans une rencontre en face à face, lorsque les thèmes de discussion viennent à manquer (de Fornel 1989 : 37-38). Le pseudonyme est aussi un signe qui individualise (ibid.): il joue à cet égard un rôle important comme procédé de mise en scène de l'identité (Marcoccia 2003). Mais comment relier pseudonyme et identité sans tomber dans l'erreur d'une mise en relation trop simpliste? Par leur pseudonyme, les usagers de chat peuvent effectivement se construire une identité imaginée, ou plutôt des identités, puisqu'il n'est pas rare qu'ils en aient plusieurs: un pseudonyme tel que <BenAfleck> (voir plus loin) est intéressant puisqu'il renvoie au monde anglo-saxon, très éloigné sur le plan géographique pour qui réside à Tananarive, mais significatif au plan symbolique. On s'attribue ainsi une personnalité internationale, donc moderne - ce qui est important pour celui qui se désigne ainsi et des jeunes qui vivent la globalisation (culturelle autant qu'économique), et le nombre important de pseudonymes anglais qu'ils choisissent est révélateur. Ces pratiques s'inscrivent dans un contexte tananarivien, où pour les jeunes urbains, les pays qui font rêver, voire qui suscitent des projets d'émigration, sont les États-Unis et le Canada. Ce dernier a remplacé la France qui ne représente plus un Eldorado: les raisons de cet abandon sont complexes, mais signalons que, contrairement aux États-Unis ou au Canada, la France est un pays largement connu par nombre d'entre eux, soit parce qu'ils y sont déjà allés étant donné qu'ils y ont de la famille, soit parce que des membres de la famille ou des amis qui résident en France viennent les visiter. La France n'est donc pas un pays inconnu sur lequel greffer tout un ensemble de représentations imaginaires, à l'instar du Canada par exemple. On trouve a contrario des usagers de chat qui affirment leur identité malgache (<ragassy>) ou préfèrent une expression en français pour se définir (<fleurdemiel>) : le choix d'un pseudonyme, sans aucun doute le résultat d'un choix personnel, s'inscrit aussi dans un ensemble de représentations liées à l'image que l'on a de soi au sein du monde social et à sa trajectoire personnelle.

\section{Les identités émergentes au cours de l'activité chat}

16 L'extrait qui suit entre <BenAfleck> et <vaza-vaseline> illustre la manière dont une histoire commune peut se construire au fur et à mesure des mouvements conversationnels. La présentation de soi dans ce type d'interactions est un indicateur fort riche : en effet, cette présentation, qui se fait de manière routinière à l'ouverture d'un chat, nous informe sur les formes d'indexation d'identités sociales, en fonction d'attentes liées à la situation. Ces routines sont révélatrices des personnes qui chattent, de la classe d'âge à laquelle elles appartiennent et des présupposés liés précisément à celle-ci. Des problèmes interactionnels qui peuvent survenir entre les participants révèlent les attentes préexistantes en termes d'identité : c'est ce que nous permet de montrer la séquence qui suit.

La personne que nous filmons - désignée dans le texte par son pseudo <BenAfleck> - est en train de chatter sur rta.org. <BenAfleck> et apparaît avoir un projet en tête, sur fond 
d'attentes préalables, que l'analyse de la séquence permet de mettre au jour. Un problème d'ordre interactionnel advient en effet dans la situation qui oblige le chatteur à expliciter ses attentes préalables d'une part, et à se repositionner d'autre part: en effet, compte tenu des répliques de son interlocuteur et des inférences qu'il peut alors établir en fonction de celles-ci, il est obligé de réviser l'orientation qu'il avait impulsée dans les premiers échanges. Nous allons montrer la manière dont s'opère ce rétablissement. Le déroulement de la séquence ${ }^{6}$ est le suivant :

«1) Séquence_<BenAfleck>_<vaza-vaseline>_rta

1. <vaza-vaseline> slt, âge ? g ou $\mathrm{f}$ ?

- (salut, (ton) âge? (tu es un) garçon (ou une) fille?

2. <BenAfleck> ben aflek ve de ho vavy na la

- NP-Ben Afleck / Q / CONJ / ?? / NC-fille / CONJ / NC-garçon

- (Ben Afleck est-ce que c'est un garçon ou une fille ?)

3. <vaza-vaseline> connais pas le malgache!

4. <BenAfleck> a bon

5. $<$ BenAfleck $>t$ vaza

- (est-ce que tu es un vazaha?)

6. <vaza-vaseline> oui c pour ca que je connis pas bien le malgache. Et toi ? tu chrerches quoi

7. <BenAfleck> une copine

8. <vaza-vaseline> pas de chance alors

9. <BenAfleck> mais t mec ?

○ (mais t'es un mec ?).»

L'ouverture de la conversation, initiée par <vaza-vaseline> (L1), avec une salutation suivie d'une demande d'information et qui concerne d'une part l'âge et le sexe de l'interlocuteur, se fait de manière routinière ainsi qu'en attestent les cas de notre corpus. Logiquement, compte tenu du fait qu'il chatte sur un site malgachophone, rta.org, <BenAfleck> pense que la personne avec qui il est en interaction est une personne malgache : ce qui nous l'indique est la réponse qu'il fait en malgache (L2) à la double requête de son interlocuteur. Pourtant, nous relevons que le pseudo de l'interlocuteur n'aurait pas dû laisser de doute quant à son identité de chatteur : <vaza-vaseline> peut se transcrire par: vazaha vaseline, où vazaha est un terme générique qui permet à une personne malgache de désigner toute personne ne ressemblant pas à un/e Malgache, le terme désignant en effet une personne à la peau claire. $\mathrm{Au}$ vu de la réplique de son interlocuteur (L3), il s'avère donc qu'effectivement la personne ne parle pas le malgache. $<$ BenAfleck> ratifie la réplique à la fois verbalement et au moyen de l'alternance codique, en passant à l'autre langue d'échange possible, le français (L4). Il demande à son interlocuteur si ce dernier est étranger (L5). Celui-ci réplique par l'affirmative (L6). La deuxième partie de sa réplique est le premier membre d'une paire adjacente qui est une question sur ce que <BenAfleck> recherche (L6). <BenAfleck> interprète cette question comme étant une demande relative au type de personne (une fille) et de relation (une copine) (L7). La réplique de <vaza-vaseline> qui arrive en troisième tour, est une évaluation négative, non pas sur le fait de répondre en termes de genre et de relation, mais sur le sexe ; ce qui entraîne <BenAfleck> à se poser en retour la question du sexe de son interlocuteur (L9).

Ce type de séquence permet de mettre au jour les attentes croisées. Lorsque celles-ci se voient contredites d'un côté comme de l'autre et que les personnes qui chattent doivent 
surmonter la désorientation qui en a résulté. <vaza-vaseline> chatte parce qu'il recherche une personne de sexe masculin ${ }^{7} ;<$ BenAfleck> quant à lui croit s'adresser à une fille, et malgache de surcroît. Si les participants s'accordent cependant sur le but de ce type d'occasions sociales - flirt ou rencontre -, en revanche, il y a méprise d'un côté sur la nature de la relation qui a présidé au contact - une relation hétérosexuelle étant recherchée par <BenAfleck>, une relation homosexuelle par <vaza-vaseline> - et, de l'autre, sur l'identité des individus, à la fois en termes d'origine (malgache ou française ?) et de genre (la plus fondamentale pour ce type d'interaction et la plus immédiatement donnée). En L8, <vaza-vaseline> exprime à la fois qu'il est de sexe masculin et donc pas l'interlocuteur approprié pour <BenAfleck>, en même temps qu'il s'adresse à lui-même cette réplique, puisque c'est lui, <vaza-vaseline> qui avait initié l'échange avec $<$ BenAfleck $>^{8}$. $<$ BenAfleck> finalement va tenter de continuer une discussion avec son interlocuteur en lui parlant de la situation (problématique) des homosexuels à Madagascar (séquence non retranscrite ici). On constate alors que les projets des individus ne changent pas seulement au cours de l'interaction, mais que ceux-ci modifient leur propre orientation quant à ce qui motivait leur prise de contact. Nous remarquons pour l'essentiel que le common ground n'est pas disponible a priori entre les participants mais qu'il s'établit au fur et à mesure que se déroule l'interaction. Cette séquence démontre qu'il est important de considérer que les identités ne sont pas fixées à l'avance mais qu'elles sont le produit des pratiques qui se modifient selon les caractéristiques même de la situation dans laquelle les personnes sont engagées. C'est dire que les interactants produisent une identité qui fait sens dans le contexte situé des actions.

\section{Les identités situées}

20 Toutefois, pour éviter une vision exclusivement « émergentiste » de l'identité, il nous faut aussi tenir compte du fait que les usagers de chat introduisent des éléments de leur propre biographie. Les chatteurs peuvent partager déjà une histoire commune, ou bien en souhaiter une, que cette histoire se limite aux conversations médiatisées ou aboutisse à une rencontre : c'est ce qui explique qu'en règle générale, les chatteurs ne s'inventent pas des personnalités et des vies imaginaires. La relation ne s'établit d'ailleurs pas exclusivement sur le pseudonyme car, très vite au début de la connexion, les «vrais » prénoms seront échangés ainsi que l'endroit où l'on se trouve au moment où l'on chatte. Cela peut déboucher éventuellement sur l'échange de numéros de téléphone avec la promesse de s'appeler, ou, pour les personnes qui sont localisées dans la même ville, une prise de rendez-vous pour se rencontrer dans un endroit donné.

21 Précisons ce thème de la localisation. Rappelons que la présupposition à l'œuvre entre usagers est que l'espace physique n'est pas partagé, ce qui explique que dans les phases d'ouverture nous trouvons toujours une séquence en plusieurs tours correspondant à une localisation réciproque. Elle est formulée sur le format suivant: en français «t'es où? ?; en malgache "aiza? " . La réponse peut concerner soit la localisation géographique dans laquelle l'interlocuteur se trouve physiquement, soit l'espace même du chat (Verdier 2009, 2010). Ces demandes relatives à la localisation s'expliquent par le fait que dans une situation standard de chat, l'accès à un espace partagé concerne uniquement la fenêtre de chat ouverte entre eux et non pas l'espace physique. 
Il est cependant toujours possible qu'une histoire biographique soit déjà partagée par les interlocuteurs, même si la connaissance qu'ils ont l'un de l'autre est le résultat d'interactions antérieures par chat et non en face à face: en ce cas, l'identité des chatteurs se précise et s'ancre en même temps dans un espace précis, du fait d'une reconnaissance mutuelle. C'est le cas de <fleurdemiel> et <ragassy> dans la séquence qui suit. Dès le début de leur conversation en privé sur wanadoo, <fleurdemiel>, après avoir salué en retour son interlocuteur, lui signale qu'elle le connaît pour avoir déjà chatté avec lui sur un autre site de messagerie. En se localisant dans la même ville, Tananarive, les interactants n'indiquent pas seulement qu'ils ont en commun une histoire interactionnelle liée au chat, mais aussi, du fait qu'ils sont tous deux des Tananariviens, qu'ils ont la connaissance d'un espace partagé, celui de la ville de Tananarive, où ils se trouvent tous deux au moment où ils chattent et qui devient le thème même de leur conversation. Nous avons déjà indiqué que nombre d'interactions par chat peuvent donner lieu à des prises de rendez-vous et il s'agit alors pour les participants de négocier un futur lieu de rencontre. C'est précisément le thème de la séquence entre <fleurdemiel> et <ragassy>. Ce dernier demande à <fleurdemiel> à quel endroit se trouve le collège où elle étudie (L1), afin de lui donner rendez-vous à un endroit qui est sur son chemin quand elle rentre chez elle (L8 \& L10). La séquence se déroule précisément de la manière suivante :

«2) Séquence_<fleurdemiel>_<ragassy>_wanadoo.mg

1. <ragassy> fa aiza ianao no mianatra ary eh ?

- CONJ / Q-où / PRON PERS 2e pers sg / CONJ / V-ACTIF-PRES-étudier / CONJ / PART VOCATIF

- (mais où est-ce que tu étudies?)

2. <fleurdemiel> square

- ([au] square) (nom d'un lycée)

3. <ragassy> aiza moa izany?

- Q-où / ?? / PRON PERS

- (c'est où ça ?)

4. <fleurdemiel> mahamasina

- (NP-Mahamasina)

- (A) Mahamasina (quartier de Tananarive, où n'est pas <fleurdemiel> à ce moment là, étant alors dans le quartier d'Isoraka)

5. <ragassy> tsy hitako anie izany eh?

- NEG / V-RAC-PRES-voir-PRON PERS $1^{\text {re }}$ pers sg / PRON PERS / PART VOCATIF

- (je ne vois pas cela!)

6. <ragassy> aiza ho aiza eo mahamasina?

- LOCUTION-où à peu près / LOCA / NP-Mahamasina

- (où ça à Mahamasina ?)

7. <fleurdemiel> fa enao (efface) (puis écrit) eo akaikiny st famille

- CONJ / PRON PERS $2^{\mathrm{e}}$ pers sg

- (mais toi)

- LOCA / ADV-près-DET / NP-Sainte Famille

- (c'est près de Sainte-Famille [Nom du collège où <fleurdemiel> étudie])

8. <ragassy> aiza ny lalanao rehefa mody ianao ?

- Q-où / DET / NC-chemin-PRON PERS $2^{\mathrm{e}}$ pers sg / ADV-quand / VACTIF-PRES-rentrer / PRON PERS $2^{\mathrm{e}}$ pers sg 
- (par où (où est ton chemin) rentres-tu ?)

9. <fleurdemiel> aty anosy

- LOCA / NP-Anosy

- ([par] [le lac] Anosy)

10. ragassy> endrasako eo @ bariera vy eo @ cnaps eo ianao hitanao ve

- V-PASSIF-attendre-PRON PERS $1^{\text {re }}$ pers sg / LOCA / PREP / NC-barrière / NC-fer / LOCA / PREP / NP-cnaps / LOCA / PRON PERS $2^{\mathrm{e}}$ pers sg / V-RAC-voir-PRON PERS $2^{\mathrm{e}}$ pers sg / Q

- (je t'attendrai à la barrière en fer près du CNAPS $^{10}$ tu vois ?)

11. <fleurdemiel> lavitra be

- ADJ-loin / ADJ-beaucoup

- (c'est loin)

12. <ragassy> fa aiza ary eh?

- CONJ / Q-où / CONJ / PART VOCATIF

$\circ$ (mais où alors?)

13. <fleurdemiel> eo @ancien com (efface)

- LOCA / PREP / NP-Anciens Combattants

- (aux anciens Combattants) (Monument près du lac Anosy). »

<fleurdemiel> écrit à <ragassy> qu'elle étudie au lieu-dit le Square (L2) dont il lui demande alors de préciser la localisation (L3). Elle lui indique le nom du quartier, Mahamasina (L4). Précisons qu'elle est dans un lieu éloigné de Mahamasina par ailleurs, située au moment de cette séquence dans le quartier d'Isoraka ainsi qu'elle l'a indiqué à son interlocuteur (voir le plan de Tananarive). Cependant, <ragassy> est incapable de situer l'endroit qu'elle désigne (L5). Il lui demande de préciser le lieu à Mahamasina (L6). Elle lui indique que le Square, le nom de son collège, est tout proche du collège Sainte Famille (L7). Il lui demande quel est le chemin par lequel elle passe pour rentrer de l'école (L8) : « par le lac Anosy » répond <fleurdemiel>. Il lui propose alors de l'attendre à la barrière en fer qui se trouve près du CNAPS (L10). À juste titre, lorsque l'on consulte le plan, <fleurdemiel> lui répond que c'est bien loin (L11). Il lui demande alors si elle a une autre idée (L12), et $<$ fleurdemiel> commence à taper une réponse, qui est une proposition de se retrouver au monument aux morts, qu'elle appelle Anciens Combattants, situé sur le lac Anosy (L13) à mi-chemin de celui que lui a proposé <ragassy> et de son collège, et par ailleurs un lieu traditionnel de rendez-vous pour les couples ainsi que nous l'avons constaté lors de notre enquête. Nous indiquons sur la carte du centre-ville de Tananarive les différentes localisations citées dans cet extrait: 


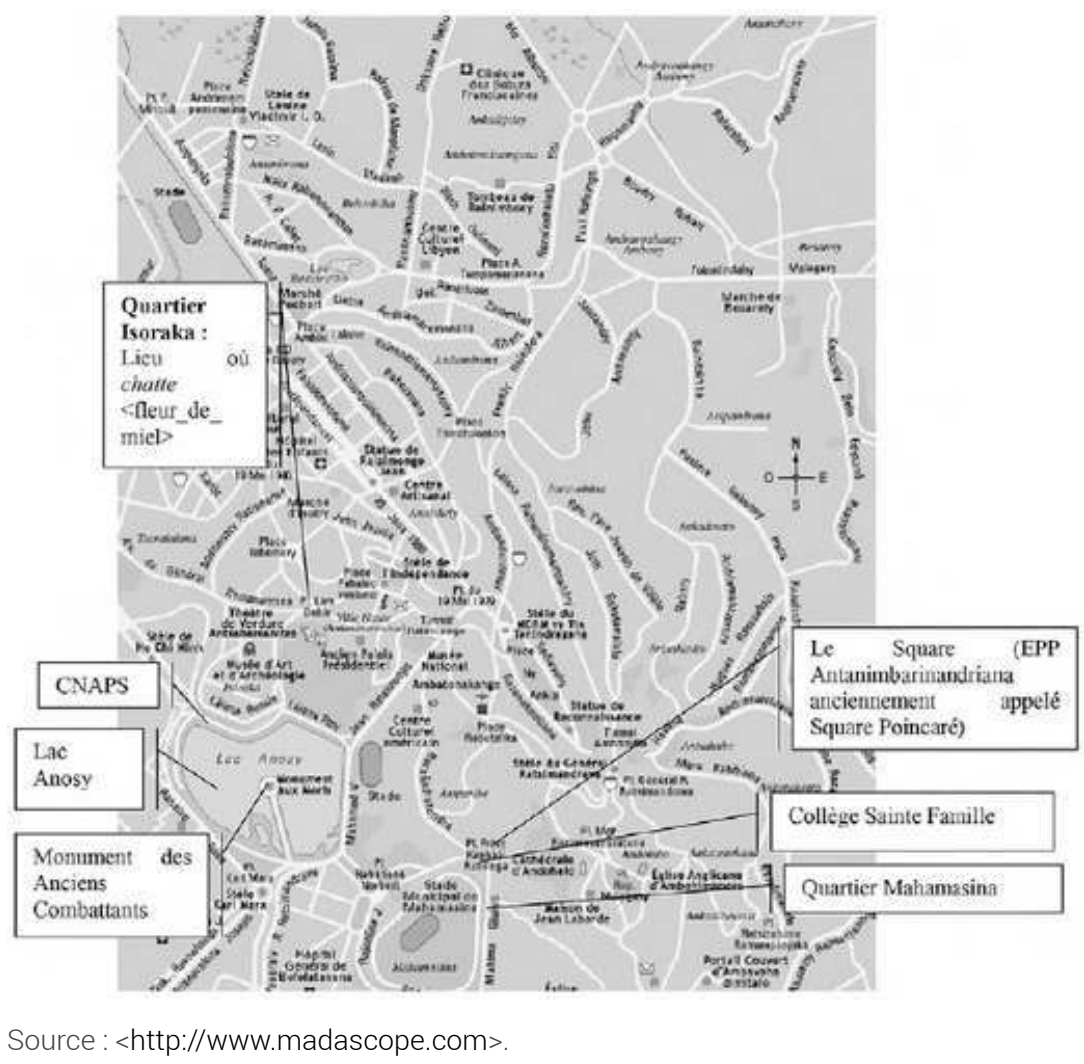

Si les interactions médiatisées par ordinateur permettent effectivement à ces jeunes Malgaches de discuter facilement avec des inconnus, elles peuvent avoir pour conséquence d'entraîner des interactions tout à fait localisées dans l'espace, en l'occurrence pour <fleurdemiel> et <raggasy>, certains quartiers de Tananarive ${ }^{11}$. Une grande importance est donc donnée à tous les éléments qui permettent d'élargir le périmètre de l'espace partagé - même de manière imaginaire. C'est dire que les interactants produisent une identité qui fait sens dans le contexte situé des actions : ils sont inévitablement conduits à référer à la fois à la situation de chat et à la situation de rencontre liée à un espace physique.

\section{La communauté de pratiques : compétences communicationnelles et répertoires partagés}

La notion d'identité nécessite de préciser l'espace social des jeunes chatteurs: la communauté à laquelle se rattachent ceux-ci dès qu'ils se connectent pour chatter inclut en effet un ensemble de pratiques supposant des compétences communicationnelles et des répertoires partagés. À la suite des travaux de M. Bucholtz (1999 : 203) dans le champ de la sociolinguistique, nous considérons en effet qu'il est préférable de faire de l'identité le produit de pratiques signant l'appartenance à une communauté sociale plutôt que de réduire cette identité à l'association d'une ipséité et d'un ensemble de traits personnels et sociaux. Les personnes s'appuient pour interagir sur un certain nombre de présuppositions liées à leurs pratiques de chat. La prise de rendez-vous entre <fleurdemiel> et <raggasy> n'a de sens que si l'un et l'autre partagent un espace de 
référence commun, ancré dans un espace géographique bien déterminé. Cette séquence révèle en outre que bien d'autres présuppositions sont à l'œuvre. Il ne fait nul doute que le critère d'âge joue un rôle essentiel entre les interactants. Ce critère détermine si une relation peut avoir lieu : quelqu'un considéré comme " trop âgé » (21 ans pour quelqu'un qui en a 17 par exemple) ou «trop jeune » (12 ans pour quelqu'un qui en a 15) peut être écarté d'emblée. Sur cette base, les participants peuvent dès lors supposer un ensemble de goûts et d'attentes partagés quant à la finalité d'une telle conversation et les sujets que l'on peut aborder (la musique, l'amour, les études notamment). Parce que <fleurdemiel> est étudiante, ainsi que le vérifie <raggasy> (L1), le lycée peut devenir un lieu de rendezvous pertinent.

Rappelons que lors d'une conversation médiatisée par ordinateur, la nature de la coprésence entre les interlocuteurs est quasi synchrone (de Fornel 1989), non pleinement partagée intersubjectivement mais ancrée spatialement dans l'écran d'ordinateur. Certes, nous l'avons $\mathrm{vu}$, les locuteurs sont physiquement inscrits dans des cadres distincts, " primaires » au sens goffmanien. Mais ils construisent bien cependant un cadre partagé par le biais de leurs répliques qui s'affichent dans la fenêtre de conversation, auquel locuteur et interlocuteur ont tous deux accès même si ce n'est que de manière partielle. Leurs répliques et l'affichage de leur pseudonyme sont les indices de la situation de coprésence (ibid. : 37). C'est donc au sein de cet espace, nullement conçu dans un registre imaginaire, qu'ils manifestent explicitement leur présence.

\section{Les compétences communicationnelles partagées}

Examinons une situation où des présuppositions à l'œuvre sont rendues explicites en raison de problèmes interactionnels: c'est le cas de la dispute entre <axara_girl> et $<$ BenAfleck $>^{12}$ dans la séquence qui suit. La première, <axara girl>, a mal interprété ce que lui a dit <BenAfleck> (non retranscrit). Pour régler ce problème interactionnel, <BenAfleck> indique d'abord qu'il n'est pas méchant (L1) et s'excuse d'avoir éventuellement fâché son interlocutrice (L3). Il effectue alors une pré-séquence consistant à vérifier si <axara_girl> aime la chanteuse Avril Lavigne (L3 et L4) - une auteure compositrice interprète pop rock canadienne. Il est lui-même en train d'écouter la chanson I'm with you de la chanteuse sur son ordinateur. Il envoie alors la réplique suivante à son interlocutrice : « je te dédit i $\mathrm{m}$ with you » (dédicace faite en français de la chanson qu'il est en train d'écouter simultanément, L4). L'action est comprise par l'interlocutrice comme l'indique sa réplique en retour (L6) en deux segments : le premier segment d'énoncé renvoie à la pré-séquence effectuée par <BenAfleck> (en L3-4) («bien sûr »); le second quant à lui est une réponse positive à l'offre de <BenAfleck> («j'adore cette chanson »):

«3) Séquence_<BenAfleck>_<axara_girl>_rta

1. $15: 16<$ BenAfleck> za tsy masika ka

- PRON PERS $1^{\text {re }}$ pers sg / NEG / ADJ-méchant / PART INTERJ

- (moi je suis pas méchant)

2. $15: 16<$ BenAfleck> dsl ra to ka nahatezitra anao

- désolé/ ADV / ADV / CONJ / V-ACTIF-PASSE-fâcher / PRON PERS $2^{\mathrm{e}}$ pers sg COMPLEMENT

- ([je suis] désolé si [je] t’ai fâchée) 
3. $15: 16<$ BenAfleck> enao ve ty avril

- PRON PERS $2^{\text {e }}$ pers sg / Q / V-RAC-aimer / NP-Avril

- (est-ce que tu aimes Avril)

4. $15: 16<$ BenAfleck> lavigne je te dedit (<BenAfleck> regarde le titre de la chanson sur son écran d'ordinateur puis continue à écrire) i m (<BenAfleck> regarde le titre de la chanson sur son écran d'ordinateur puis continue à écrire) with you (et il envoie le tour d'écriture suivant :)

- lavigne je te dedit i $m$ with you

- NP-Lavigne / je te dédie «I am with you » (le titre d'une chanson de Avril Lavigne)

- (Lavigne / je te dédie « I m with you »)

5. (<BenAfleck> passe à une autre fenêtre de conversation chat - non retranscrit ici - puis il revient à sa conversation avec <axara_girl $>$ )

6. 15 :18 <axara_girl> b1 sur j'ador ct chanson

- (bien sûr j'adore cette chanson)

7. $15: 18<$ BenAfleck> za tena siba be aminy

- PRON PERS $1^{\text {re }}$ pers sg / ADV-vraiment / ADJ-fou / ADJ-très / ADV-3 ${ }^{\text {e pers sg }}$

- (moi j'en suis vraiment fou)

8. $15: 18<$ BenAfleck> tsy izy rery fa sa mikif punk be de mirevy avril la princesse

- NEG / PRON PERS $3^{\text {e }}$ pers sg / ADV-seulement / CONJ / PRON PERS $1^{\text {re }}$ pers sg / VACTIF-kiffer / NC-punk / ADJ-très / CONJ / V-ACTIF-rêver / NP-Avril la princesse

- (mais pas seulement elle, je kiffe beaucoup le punk et je rêve d'Avril la Princesse). »

Les photos qui suivent ${ }^{13}$ illustrent ce segment d'interaction, en montrant (photo 1) les regards que jette <BenAfleck> sur son propre écran pour vérifier la manière dont s'écrit le titre de la chanson d'Avril Lavigne qu'il est en train d'écouter à l'instant même sur son ordinateur; puis (photo 2), le moment où il vient d'envoyer son tour (L4). 
ILL. 5. <BENAFLECK> A COMMENCÉ À ÉCRIRE DANS LA ZONE DE SAISIE CE QUI N'EST PAS VISIBLE POUR SON INTERLOCUTRICE, « LAVIGNE JE TE DEDIT » PUIS IL REGARDE LE TITRE DE LA CHANSON SUR SON ÉCRAN D'ORDINATEUR AVANT DE CONTINUER À ÉCRIRE

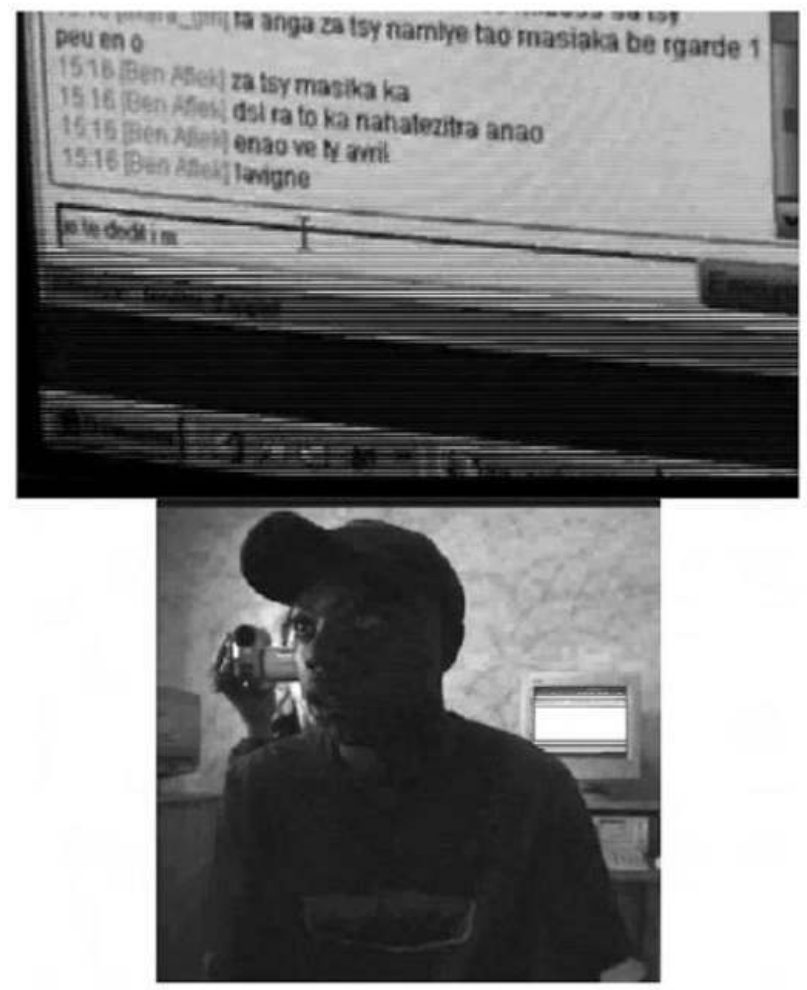

ILL. 6. <BENAFLECK> A FINI D'ÉCRIRE ET A ENVOYÉ SON TOUR D'ÉCRITURE À <AXARA_GIRL> « LAVIGNE JE TE DEDIT I M WITH YOU » QUI APPARAÎT ALORS DANS L'ESPACE DE CONVERSATION PRIVÉE QU'IL PARTAGE AVEC <AXARA_GIRL>

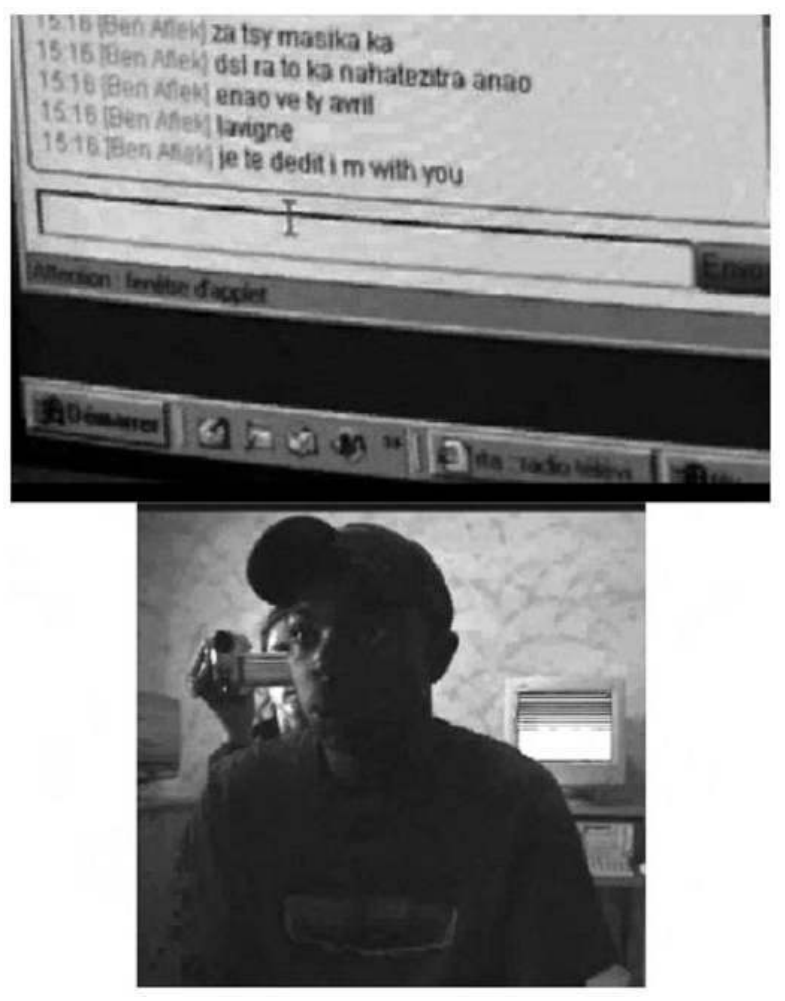



alors même que <BenAfleck> et <axara girl> ne partagent pas pleinement le même espace perceptif (et notamment auditif) étant donné que la situation de coprésence est établie via l'inscription de leurs tours d'écriture dans une fenêtre d'ordinateur, <BenAfleck> peut-il faire l'action d'offrir quelque chose à son interlocutrice qui n'est pas disponible pour elle? Et comment <axara_girl> peut-elle comprendre une telle offre et donc y répondre favorablement? Que l'espace intersubjectif ne soit que partiellement partagé n'exclut pas que les participants puissent articuler ce qui se passe dans leur cadre physique avec ce cadre conversationnel: ainsi l'action d'offrir de <BenAfleck> repose précisément sur cette possibilité qu'ont les chatteurs d'inclure dans l'espace conversationnel chat des éléments de leur espace physique. Cette possibilité est admise par tous les interactants. L'action de <BenAfleck> ne peut donc être comprise de <axara_girl> que si celle-ci partage avec son interlocuteur cette donnée de base: les cadres ne sont pas étanches. Si <axara_girl> répond favorablement à l'offre de dédicace, c'est sans nul doute parce qu'elle sait que des éléments du contexte physique dans lequel l'autre participant est inscrit peuvent être mobilisés comme ressource conversationnelle. Exploiter activement cette relation de coprésence permet de pallier le fait qu'une condition de félicité de l'acte, à savoir l'existence d'un espace auditif partagé, n'est pas remplie. La réussite de l'acte performatif - qui est reconnu comme tel par l'interlocutrice - et l'existence d'effets perlocutoires (rétablir une entente entre les interactants) reposent sur le fait que pour les interactants la situation est une situation de coprésence ${ }^{14}$

L'action performative réussit aussi parce que <BenAfleck> et <axara_girl> partagent un certain nombre de représentations communes qui, cette fois, ne sont plus liées à la situation de chat mais à leur histoire sociale commune en lien avec leur âge (<BenAfleck> a 19 ans, <axara_girl> 21 ans) : où écouter de la musique pop rock (plutôt que de l'opérette) va de soi ; où l'on considère que ce type de musique "adoucit les mœurs " (malgré le rythme énergique); où "envoyer une dédicace » est une activité qui a du sens et fait partie des ressources disponibles pour régler un problème interactionnel (comme c'est le cas dans certaines émissions de radio qu'ils écoutent par ailleurs); où Avril Lavigne est un nom (re)connu ; où écrire « je te dedit i $m$ with you » signifie quelque chose, c'est-à-dire : a) que l'interlocutrice comprend qu'il s'agit de l'action de dédicacer; b) qu'elle a identifié qu'il y avait deux langues différentes, et que pour elle, l'alternance du malgache au français, puis à l'intérieur même d'un énoncé du français à l'anglais est possible, et est même la norme ; c) qu'elle est capable de comprendre que «I $\mathrm{m}$ with you » est le titre d'une chanson... et l'on pourrait multiplier ainsi les conditions nécessaires pour que l'interaction réussisse. S'ils arrivent à se comprendre, c'est qu'ils appartiennent à la même communauté de pratiques et qu'ils partagent aussi un certain nombre de présupposés liés à leur classe d'âge, au fait aussi d'être malgaches et d'habiter en ville - à l'époque de notre enquête, chatter est surtout une activité de jeunes urbains.

\section{Les répertoires partagés}

31 Chatter suppose donc des compétences communicationnelles, comme nous venons de l'évoquer, et des répertoires partagés. Attardons-nous maintenant sur ce deuxième point. La séquence précédente avec <BenAfleck> illustre la possibilité d'alterner entre différentes langues, voire même de les mélanger, lorsque l'on chatte : <axara_girl> répond

Cahiers d'études africaines, 215 | 2014 
en français (L6) à son interlocuteur qui lui-même associe malgache et français (L8). Code switching et code mixing ${ }^{15}$ sont des phénomènes communs dans les situations d'écrit médiatisé (Lexander 2007 pour les situations incluant le français, McIntosh 2010 pour le Kenya). En outre, les phénomènes d'écrit non conventionnel y sont largement répandus : les termes, qu'ils soient écrits en français ou en malgache ne le sont pas de manière conventionnelle. Comment comprendre de telles pratiques qui, par ailleurs, ne sont pas particulières à la situation malgache?

Dressons rapidement l'arrière-plan sociolinguistique de ces jeunes chatteurs. Tous ceux que nous avons rencontrés ont eu au moins le malgache officiel comme langue maternelle, langue enseignée et langue d'enseignement; et tous ont eu accès au français comme langue enseignée et langue d'enseignement; pour quelques-uns d'entre eux, le français peut être parlé par l'un des parents à la maison. Étant très présent dans les médias, le français ne se limite pas à des contextes scolaires. Tandis qu'ils sont exposés quotidiennement au français (école, médias), pour lequel ils développent une compétence au moins passive, le malgache reste en revanche, pour les jeunes que nous avons rencontrés, la langue des échanges quotidiens, entre pairs ou en famille, ainsi que celle des situations officielles (rituelles). Bien que le malgache, langue officielle du pays, soit utilisé dans l'enseignement, par les médias et dans la vie politique et culturelle, au sein de l'administration centrale, on écrira plus fréquemment le français que le malgache officiel. Malgré la période de malgachisation (1973-1992), avec un enseignement en malgache, le français demeure encore le premier instrument de promotion sociale (Rabenoro \& Rajaonarivo 1997).

Nous avons déjà souligné que les variables sociales sont loin d'être neutralisées dans ce type d'échange: cela vaut aussi pour les échanges linguistiques. Interrogée sur ses pratiques de communication, <Anta>, une jeune fille de 16 ans, évoque ce qu'elle ressent face à un interlocuteur qui chatte en français ou en malgache :

«<Anta> : ouais pour moi c'est personnel je sais pas pour moi il parle en français ça veut dire que c'est pas un garçon n'importe qui qu'il a quand même atteint un certain niveau d'étude donc pour moi c'est pour ça qu'il parle en français s'il parle en malgache je sais pas pour moi eh ben je me dis peut-être qu'il ne sait pas parler en français ou je sais pas

M. Verdier : d'accord c'est aussi une manière de savoir qui il est s'il a fait des études $<$ Anta> : et puis ou bien il fréquente quel genre de personne parce qu'on voit à travers le langage qu'il utilise aussi parce qu'il y a ceux qui disent de gros mots en malgache et c'est pas bien aussi ».(entretien avec <Anta>, Tananarive 2005)

L'utilisation du français, du malgache et du variaminanana indifféremment pour échanger ne signifie donc pas pour autant que les rapports de force entre les langues soient complètement renversés ou même annulés.

Qu'en est-il de l'écrit du chat maintenant ? Il s'écarte de l'écrit standardisé et présente de nombreux effets - graphiques notamment. Que les personnes soient malgachophones ne change rien à la nature de leurs écrits qui ont les mêmes caractéristiques que l'on peut rencontrer dans les chats écrits en français ou dans d'autres langues ${ }^{16}$. Ces phénomènes sont par ailleurs connus et étudiés (Pierozak 2000), étant la marque même de ces situations. Celles-ci supposent en effet un type d'écrit qui doit avoir les marques de l'oral et qui doit être rapide parce que la réussite de l'interaction est à ce prix. Nous avons montré ailleurs (Verdier 2013) que, dans le cas malgache, il n'y a pas de langue d'échange préférée dans ces situations: les chatteurs, s'ils interagissent avec une personne malgachophone, utilisent le français, le malgache et le variaminanana (mélange de 
français et de malgache) ${ }^{17}$. Il n'est pas rare, à l'instar de l'exemple entre <BenAfleck> et <axara_girl>, qu'ils commencent à chatter en français puis continuent en malgache. Parce qu'ils sont dans une situation informelle, les chatteurs ne sont pas sous la coupe des discours dominants visant à stigmatiser le mélange de langues - ni dans le sens d'une attitude de révolte, ni dans le sens d'une internalisation de ces discours qui entrainerait une dévalorisation de leur pratique (Jones \& Schieffelin 2009 : 32-33). Conséquence : ils écrivent sans la pression de la norme dominante, ce qui explique que l'on trouve des termes malgaches et français, alors même que les locuteurs peuvent ne pas maîtriser parfaitement l'une des deux langues.

Soulignons combien la situation serait pour le moins incongrue si elle avait lieu dans une situation de face à face, où la langue d'interaction serait le malgache. En effet, à moins d'y être très à l'aise (et notamment de ne pas avoir d'«accent»), les jeunes Malgaches n'interagissent pas facilement entre eux en français. Pour en rendre compte, partons d'une situation particulière, mais cependant plus répandue que l'on ne le croit, en tout cas à Tananarive : il s'agit d'un cas où le cadre physique se trouve coïncider avec le cadre interactionnel focalisé, ou bien, en d'autres termes, où deux personnes qui font connaissance en chattant sur rta.org se trouvent en fait à deux pas l'un de l'autre dans le même cybercafé ${ }^{18}$. La séquence concerne un usager, <Aly bemoukony>, et deux chatteuses qui utilisent un seul pseudo, <kokine>. <Aly bemoukony> chatte sur le site de rencontre rta.org, avec son frère assis à côté de lui, qui surfe lui aussi sur l'Internet.

ILL. 7. <ALY BEMOUKONY> SE RETOURNE VERS LES DEUX JEUNES FILLES, ASSISES NON LOIN DE LUI, QUI CHATTENT SOUS LE PSEUDO <KOKINE> AVEC LUI SUR RTA.ORG

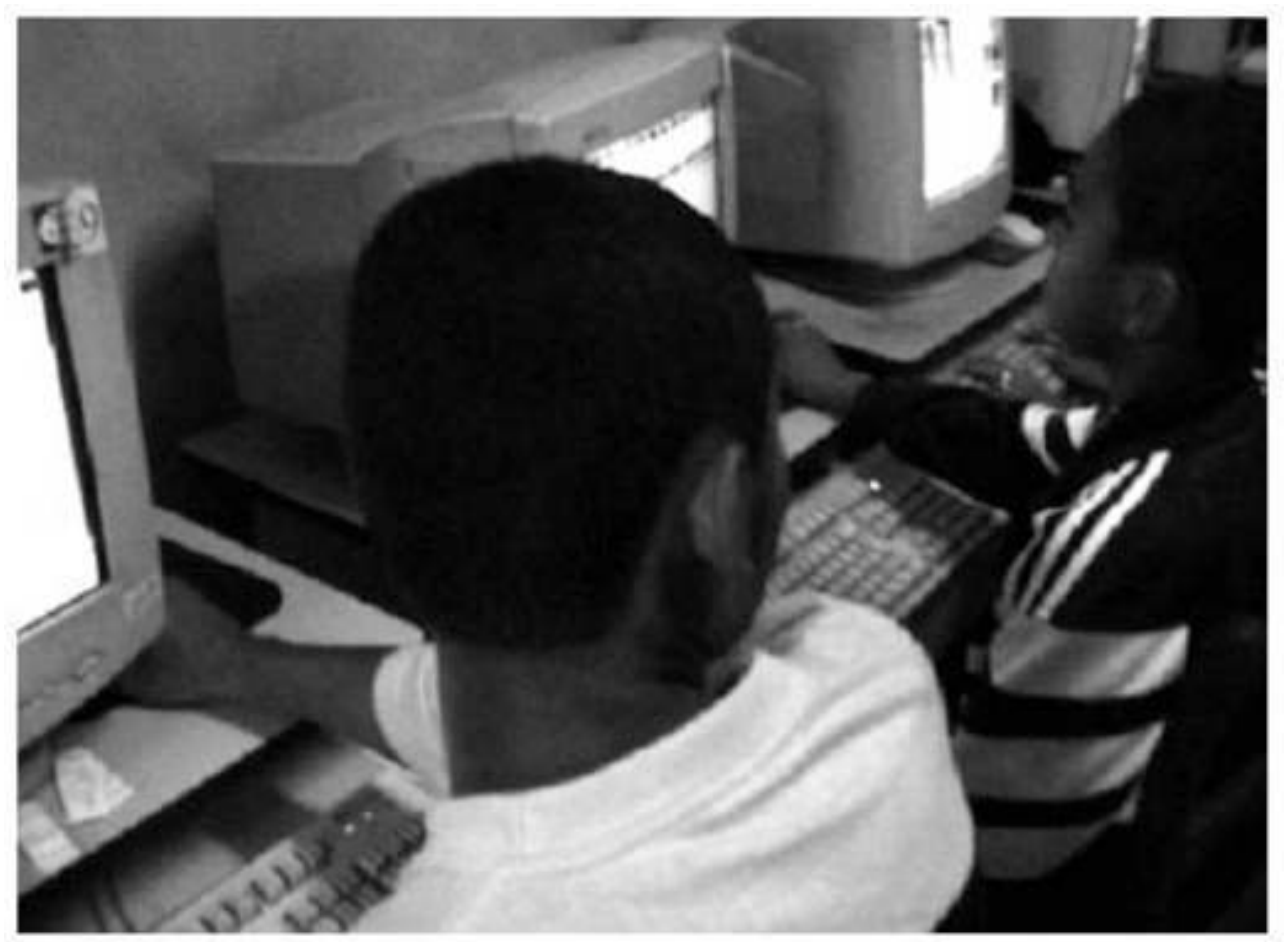

37 Assis à côté de <Aly bemoukony>, son frère se rend compte que les deux jeunes filles derrière eux sont elles aussi connectées sur rta. De sa place, il peut voir leur pseudo, $<$ kokine>, qu'il indique à son frère. Ce dernier ouvre alors une discussion privée («pv») avec elles, indiquant dès l'ouverture : «vous êtes dans le cyber de l'ouest, non?» À un 
certain moment, <Aly bemoukony> se retourne pour les regarder; puis il regarde la fenêtre de chat privé ouverte sur son écran où s'affiche la conversation qu'il a avec $<$ kokine>. Celle-ci lui a répondu : «tu es où ? »<Aly bemoukony> répond alors : «je vous dis que je suis dans le cyber de l'ouest. » Nous attirons l'attention vers le pronom utilisé par <Aly bemoukony> : il ne peut s'expliquer si l'on regarde seulement l'interaction chat parce que rien dans cette interaction ne lui indique que <kokine> renvoie à deux personnes; et compte tenu du fait que le chat implique des jeunes gens et suppose une relation de connivence, il ne peut s'agir de l'emploi du vouvoiement qui serait inapproprié. L'utilisation de ce pronom s'explique par le fait qu'il réfère à ce qu'il a perçu dans le cadre primaire. Nous observons par ailleurs que jamais, ni lui ni les deux jeunes filles ne se lèvent pour venir se parler durant la session de chat: pendant toute la durée de celle-ci, ils restent focalisés sur les interactions à l'écran. Lorsque la connexion chat se termine, <Aly bemoukony> quitte son ordinateur et vient saluer la plus âgée des deux jeunes filles. Alors qu'il interagissait en français, le jeune garçon salue ses nouvelles relations en malgache : le français serait inhabituel entre malgachophones dans le cadre d'une rencontre en face à face, d'autant plus qu'il n'est pas un locuteur très à l'aise en français ainsi qu'il nous le laisse entendre durant l'entretien que nous avons avec lui. Il continue à parler en malgache par la suite lorsqu'il leur explique pourquoi il est filmé et qui nous sommes.

Si nous observons que le statut des langues est modifié dans les interactions médiatisées par ordinateur, nos analyses montrent néanmoins que l'espace des interactions médiatisées par chat est un micro-champ où les rapports de forces entre les langues s'atténuent, mais ne sont pas neutralisés et ce parce que l'espace des conversations chat s'inscrit dans un espace de conversations plus large ${ }^{19}$. Dans les interactions médiatisées, un certain nombre de traits non maitrisés par le locuteur malgachophone s'exprimant en français, ce qui pourrait le stigmatiser (et donc le rendre muet), disparaissent: citons, entre autres, l'accent, une syntaxe approximative, la difficulté à trouver le mot juste et, pour la dimension écrite, l'orthographe. Cependant, même si le français est utilisé dans les chats sur le même plan que le malgache, son emploi apporte sans nul doute des profits symboliques (comme le montre l'entretien avec <Anta). Postuler a priori que les situations de chat sont des espaces d'innovations linguistiques et identitaires consiste à ignorer la nature même de ces interactions qui, loin d'être des espaces clos et coupés du champ social, s'inscrivent au contraire dans l'économie plus générale des échanges langagiers.

Si l'on opte pour une vision située des identités dans les interactions médiatisées, on se rend compte que les indices socioculturels, présents dans une relation de coprésence, ne disparaissent pas pour autant dans les interactions médiatisées. L'échange est nécessairement porteur d'indices. Que penser de l'argument selon lequel les espaces numériques sont l'occasion pour les personnes de se créer de nouvelles identités? L'identité est alors conçue comme une performance avec au fond une critique de la conception classique d'une identité comme quelque chose de stable et permanent. En montrant la nécessité qu'il y a à s'extraire de l'opposition réel/virtuel pour penser les identités émergentes dans les interactions médiatisées et en nous appuyant sur l'observation des pratiques, nous avons montré la nature incarnée de l'activité des chatteurs et opté pour une vision plutôt hybride de la notion d'identité. En partant de la notion d'agent social avec une identité en conséquence elle-même située, nous avons 
montré que les identités ne sont pas fixées à l'avance: elles se modifient selon les caractéristiques même de la situation dans laquelle les personnes sont engagées. Loin de confirmer l'existence d'une opposition entre virtuel et réel, les séquences étudiées ont alors permis d'observer que s'interpénètrent les relations que l'on souhaite établir et les relations déjà établies qui peuvent rester de l'ordre du chat ou se transformer en relations de face à face. Les relations que les jeunes établissent lorsqu'ils chattent sont certes délocalisées - puisque médiatisées entre des personnes qui ne partagent pas le même espace intersubjectif - mais peuvent néanmoins à tout moment être relocalisées compte tenu des connaissances partagées. Soulignant l'importance de l'ancrage des chatteurs observés dans les cybercafés de Tananarive dans un ici et maintenant, nous avons montré qu'il y a bien en un sens un mode d'existence virtuelle, du fait de l'usage des pseudonymes notamment, qui crée une sorte d'identité liée à la pratique même du chat, et qu'il existe donc peut-être, du fait de connections répétées, un effet de communauté virtuelle. Mais cela n'implique nullement une séparation définitive entre deux types d'identité sociale selon que les personnes interagissent médiatiquement ou en face à face. Parce qu'ils partagent une communauté de pratiques et de répertoires, ces chatteurs peuvent arriver à se comprendre et à interagir. Cette communauté est le produit, d'une part, de connexions répétées - ce qui est largement considéré dans les études sur les nouvelles technologies - et, d'autre part, et cela est plus rarement souligné et sur quoi nous insistons, de ce que les usagers de chat s'ancrent bien dans un monde social (Jones \& Schieffelin 2009) : ils appartiennent plus ou moins à la même classe d'âge, habitent même quelquefois la même ville, partagent tout à la fois un ensemble de comportements verbogestuels et ont aussi en commun des goûts, des intérêts et des représentations. S'il est vrai que les interactions médiatisées par ordinateur tendent à effacer certains signes tangibles d'une appartenance à un certain groupe social, comme la manière de s'habiller, de parler, de bouger, tous les indices ne disparaissent pas pour autant. Les personnes qui interagissent par l'Internet sont pourvues de qualités stables parce qu'elles sont des êtres sociaux. Étant donnée la nature même de l'activité de chat, qui fait qu'on est amené à développer des thèmes de conversation par exemple sur le lieu où l'on se trouve et sur la nature de la relation, des indices socioculturels sont inévitablement réintroduits dans les conversations. Plus généralement, le jeu des éléments sociaux qui structurent toute interaction se trouve réactivé et transformé.

Plus nous avons progressé dans l'analyse des interactions médiatisées et plus il s'est avéré indispensable de considérer que les concepts d'identité et de communauté, pour être des concepts opératoires pour une analyse anthropologique des interactions médiatisées, doivent être définis en tenant compte, d'une part, de la nature de l'interaction et, d'autre part, de la nature incarnée des pratiques langagières.

\section{BIBLIOGRAPHIE}

AUER, P., 2013, Code-Switching in Conversation, Language Interaction and Identity, London, Routledge. 
AXEL, B. K., 2006, «Anthropology and the New Technologies of Communication », Cultural Anthropology, 21 (3) : 354-384.

BABAULT, S., 2003, «Plurilinguisme et tensions identitaires chez les lycéens malgaches », Glottopol, $2: 134-146$.

Bargh, J. A, mckenna, K. Y. A. \& fitzsimons, G. M., 2002, « Can You See the Real Me ? Activation and Expression of the "True Self" on the Internet », Journal Sociological Issues, 58 (1) : 33-48.

BARON, N. S., 1984, «Computer Mediated Communication as a Force in Language Change », Visible Language, XVIII (2) : 118-141.

BARON, N. S., 2004, « See You Online : Gender Issues in College Student Use of Instant Messaging », Journal of Language and Social Psychology, 23 (4) : 397-423.

BAvouX, C., 2000, Le français de Madagascar. Contribution à un inventaire des particularités lexicales, Bruxelles, Duculot.

BAYM, N., 1995, " The Emergence of Community in Computer-Mediated Communication », in S. JONES (ed.), Cyber Society, Newbury Park, CA, Sage : 138-163.

BEAUdouin, V. \& VELKovsKa, J., 1999, « Constitution d'un espace de communication sur Internet (forums, pages personnelles, courrier électronique...) ", Réseaux, 97 : 121-177.

BOURDIEU, P., 1980, Questions de sociologie, Paris, Éditions de Minuit.

BuCholtz, M., 1999, " “Why Be Normal ?" : Language and Identity Practices in a Community of Nerd Girls », Language in Society, 28 (2) : 203-223.

BYRNE, E., 1994, The Formation of Relationships on Internet Relay Chat (IRC), Bachelors of Arts (Honors), Applied Communication Studies, Sydney, University of Western Sydney, Faculty of Humanities and Social Sciences.

COOK, S., 2004, « New Technologies and Language Change : Toward an Anthropology of Linguistic Frontiers ", Annual Review of Anthropology, 33 : 103-115.

CORRELL, S., 1995, « The Ethnography of an Electronic Bar », Journal of Contemporary Ethnography, $24: 270-298$.

CRYSTAL, D., 2004, Language Revolution, Cambridge, Polity Press.

ESCOBAR, A., 1994, « Welcome to Cyberia : Notes on the Anthropology of Cyberculture », Current Anthropology, 35 (3) : 211-231.

FERRARA, K., BRUNNER, H. \& WHITTEMORE, G., 1991, « Interactive Written Discourse as an Emergent Register ", Written Communication, 8 (1) : 8-34.

DE FORNEL, M., 1989, « Une situation interactionnelle négligée : la messagerie télématique », Réseaux, $38: 31-48$.

GARFINKEL, H., 2006, Seeing Sociologically: The Routine Grounds of Social Action, Boulder, Co \& London, Paradigm Publisher.

GEORGES, F., 2011, « L'identité numérique sous l'emprise culturelle. De l'expression de soi à sa standardisation ", Les cahiers du numérique, $1: 31-48$.

HANKS, W. F., 1996, Language and Communicative Practices, Boulder, Co, Westview Press. 
HARAWAY, D., 1991, «A Cyborg Manifesto : Science, Technology, and Socialist-Feminism in the Late Twentieth Century », in D. HARAWAY (ed.), Simians, Cyborgs and Women : The Reinvention of Nature, New York, Routledge : 149-181.

HERRING, S. C., JOHNSON, D. A. \& DI BENEDETTO, T., 1992, « Participation in Electronic Discourse in a "Feminist" Field ", in Locating Power : Proceedings of the 1992 Berkeley Women and Language Conference , Berkeley, Berkeley Women and Language Group : 250-262.

JONES, S., 1998, CyberSociety 2.0 : Revisiting Computer-mediated Communication and Community, Newbury Park, CA, Sage Publications.

JONES, G. M. \& SCHIEFFELIN, B. B., 2009, « Enquoting Voices, Accomplishing Talk : Uses of be + like in Instant Messaging ", Language \& Communication, 29 (1) : 77-113.

KEATING, E. \& MIRUS, G., 2003, « American Sign Language in Virtual Space : Interactions between Deaf Users of Computer-Mediated Video Communication and the Impact of Technology on Language Practices », Language in Society, $32: 693-714$.

KIESLER, S. \& SPROULL, L. (EDS.), 1987, Computing and Change on, Cambridge, Cambridge University Press.

KOZINETS, R. V., 2002, « The Field Behind the Screen : Using Netnography for Marketing Research in Online Communities », Journal of Marketing Research, 39 (1) : 61-72.

LEXANDER, K. V., 2007, « Langues et SMS au Sénégal. Le cas des étudiants de Dakar », in J. GERBAULT (dir.), La langue du cyberespace. De la diversité aux normes, Paris, L'Harmattan.

MARCocciA, M., 2003, « Parler politique dans un forum de discussion », Langage et société, 104 : 9-55.

MARKHAM, A., 1998, Life Online : Researching Real Experiences in Virtual Space, Walnut Creek, CA, AltaMira Press.

MARKHAM, A., 2013, «Fieldwork in Social Media : What Would Malinowski Do?», Qualitative Communication Research, 2 (4) : 434-446.

Mcintosh, J., 2010, « Mobile Phones and Mipoho's Prophecy : The Powers and Dangers of Flying Language ", Journal of the American Ethnological Society, 37 (2) : 337-353.

MCKENNA, K. Y. A., GREEN, A. S. \& GLEASON, M. J., 2002, « Relationship Formation on the Inter-net : What's the Big Attraction? », Journal of Social Issues, 58 (1) : 931.

MURRAY, D. E., 1988, « Computer-mediated Communication : Implications for ESP », English for Specific Purposes, 7 : 3-18

PASTINELLI, M., 2011, « Pour en finir avec l'ethnographie du virtuel ! Des enjeux méthodologiques de l'enquête de terrain en ligne ", Anthropologie et Sociétés, 35 (1-2) : 35-52.

PIEROZAK, I., 2000, « Approche sociolinguistique des pratiques discursives en français sur Internet : "ge fé dais fotes si je vœux" ", Revue Française de Linguistique Appliquée, 5 (1) : 89-104. POSTER, M., 1990, The Mode of Information : Poststructuralism and Social Context, Chicago, University of Chicago Press.

RABENORO, I. \& RAJAONARIVO, S., 1997, «À l'aube du 21 e siècle, quelle politique linguistique pour Madagascar?», Politiques linguistiques, $52: 105-119$.

RASOLONIAINA, B., 2003, « Le variaminanana des marchands de Tanjombato, zone rurbaine d'Antananarivo », Glottopol, 2 : 108-118. 
RAWLS, A. W., 2005, « Garfinkel's Conception of Time », Time \& Society, 14 (2-3) : 163-190.

SANKOFF, G. \& POPLACK, S., 1981, « A Formal Grammar for Code-Switching », Papers in Linguistics, $14(1): 346$.

TURKLE, S., 1995, Life on the Screen : Identity in the Age of the Internet, New York, Simon \& Schuster Ed.

VERDIER, M., 2009, « Deixis ad oculos et deixis à l'imaginaire dans les interactions médiatisées par ordinateur en malgache ", in J. FRIEDRICH (dir.), Verbum, Nancy, Presses universitaires de Nancy : 153-176.

VERDIER, M., 2010, Le Temps des échanges. Anthropologie linguistique des chats dans les cybercafés de Tananarive (Madagascar), Thèse de doctorat, Paris, École des hautes études en sciences sociales. VERDIER, M., 2013, « La constitution de l'idéologie linguistique des chatteurs malgachophones dans les cybercafés de Tananarive », Langage \& société, 143 (1) : 87-107.

WERRY, C. C. \& MOWBRAY, M. (DIR.), 2001, Online Communities: Commerce, Community Action \& the Virtual University, Upper Saddle River, NJ, Prentice Hall.

WHEELER, D., 2001, « New Technologies, Old Culture », in C. ESS (dir.), Culture, Technology,

Communication, Albany, State University New York Press : 187-212.

WILSON, S. M. \& PETERSON, L. C., 2002, « The Anthropology of Online Communities », Annual Review of Anthropology, $31: 449-467$.

WOOLARD, K., 2004, « Codeswitching », in A. DURANTI (ed.), A Companion to Linguistic Anthropology, Oxford, Blackwell Publishing : 73-94.

ZAGALO, N., MORGADO, L. \& BOA-VENTURA, A., 2012, Virtual Worlds and Metaverse Platforms : New Communication and Identity Paradigms, Information Science Reference (an imprint of IGI Global), Hershey PA, USA.

ZHAO, S., GRASMUCK, S. \& MARTIN, J., 2008, « Identity Construction on Facebook : Digital

Empowerment in Anchored Relationships », Computers in Human Behavior, 24 : 1816-1836.

\section{NOTES}

1. Nous partons de la notion d'agent social, i.e. un être doté de compétences pratiques lui permettant de participer et de s'engager dans des activités situées, avec une identité en conséquence elle-même située (RAWLS 2005 ; GARFINKEL 2006).

2. Cette recherche a bénéficié d'une aide du Programme interdisciplinaire CNRS « Traitement des connaissances, apprentissage et NTIC (2004-2006).

3. Le chat de type mIRC, qui utilise le serveur de l'Internet Relay Chat (IRC), permet de discuter avec d'autres personnes connectées dans des «chat rooms» («salons de discussion »).

4. Chaque site de rencontre chat a plus ou moins ses habitués et des spécificités que nous discutons ailleurs (VERDIER 2010).

5. Noter que ceci est exact dans les cas des sites de rta.org et wanadoo.mg, mais pas de tous les sites de chat consultés par les internautes malgaches rencontrés ni non plus du logiciel de messagerie instantanée Yahoo Messenger. 
6. Les séquences de chat présentées dans cet article se lisent de la manière suivante : (1) les pseudos des chatteurs écrits entre $>$ en début de ligne numérotée indiquent la personne à l'origine de la prise de tour d'écriture ; (2) (2a) la première ligne numérotée correspond aux échanges par chat; (2b) la deuxième ligne est une traduction libre de l'échange en malgache ou en français; (2c) lorsque l'énoncé est en malgache, nous intercalons entre les deux lignes une transcription au mot à mot où nous indiquons les catégories grammaticales qui nous servent à titre descriptif.

7. Le terme vaseline indique qu'il recherche des relations d'un certain type, ce que ne relève pas <BenAfleck>.

8. Nos données audiovisuelles l'attestent.

9. "aiza » est un adverbe locatif (substitut locatif) apte à la fonction prédicative et à la fonction de sujet postposé d'un prédicat non défini. Il signifie « où ? » en français.

10. CNAPS : Caisse nationale de prévoyance sociale (voir sur le plan de Tananarive).

11. Pour une analyse des déictiques employés dans cette séquence, voir M. VERDIER (2009).

12. Nous avons filmé <BenAfleck> en train de chatter dans un cybercafé de Tananarive.

13. Les deux angles de vue ont été synchronisés.

14. On notera que le titre anglais «I $\mathrm{m}$ with you » signifie précisément la coprésence. On aurait pu analyser l'acte de <BenAfleck> au niveau du signifiant seulement de la chanson : il envoie une chanson dont le titre est « je suis avec toi » pour signifier qu'il est bien avec elle. Une telle analyse est impossible compte tenu de nos données et des connaissances contextuelles que nous avons : il envoie cette chanson parce qu'il est justement en train de l'écouter (il vérifie l'écriture du titre par deux coups d'œil à l'écran) et qu'elle correspond précisément à l'ambiance qu'il veut instaurer avec son interlocutrice - et non pas en raison du titre (nous savons que <BenAfleck> ne connaît pas suffisamment l'anglais pour que ce soit la signification du titre qui joue en premier lieu).

15. Pour ces questions, consulter G. SANKOFF et S. POPLACK (1981), K. WOOLARD (2004), P. AUER (2013).

16. Ils présentent toutefois des variations intéressantes à étudier selon qu'ils soient écrits en malgache ou en français (VERDIER 2013).

17. Pour une étude du variaminanana, voir S. BABAUlt (2003), C. BAvoux (2000), B. RASOLONIAINA (2003), M. VERDIER (2013).

18. Le cas est un peu plus fréquent qu'on ne pourrait le supposer a priori dans les situations observées à Tananarive - plusieurs cas similaires ont été rapportés à l'auteure de cette recherche qui, pour sa part, a chatté à plusieurs reprises avec une personne présente dans le même cybercafé en le sachant (et en le faisant savoir).

19. P. BOURDIEU (1980: 124) explique ainsi que « toute communication linguistique, même entre deux personnes, entre deux copains, entre un garçon et sa petite amie, toutes les interactions linguistiques sont des espèces de micromarchés qui restent toujours dominés par les structures globales ». 


\section{RÉSUMÉS}

À partir de l'étude anthropologique des pratiques de chat dans les cybercafés de Tananarive, on remet en cause l'idée largement répandue que la médiatisation des interactions entraîne obligatoirement la création de nouvelles identités sociales évoluant dans des communautés virtuelles. Nous étudions dans un premier temps la manière dont les identités entre jeunes chatteurs se construisent tout à la fois dans les chats et dans un environnement géographiquement et socialement situé. Dans un deuxième temps, nous montrons que les interactions médiatisées par ordinateur s'inscrivent aussi dans un espace social que l'on peut caractériser anthropologiquement, ce qui a des effets non négligeables sur les échanges; inversement, les chatteurs s'appuient pour interagir sur un certain nombre de présuppositions liées à leurs pratiques de chat.

Identities and Community of Practices of the Malagasy Users of Mediated Interactions in the Cybercafés of Antananarivo (Madagascar)

Based on an anthropological study of computer mediated interactions in the cybercafés of Antananarivo, this article challenges the idea that the practices of CMO necessarily lead to the creation of new social identities. Instead we argue that identities are blended with identities anchored in a certain environment located geographically and socially. Rather than assuming that people have a virtual identity in a virtual community, we emphasize that computer mediated interactions are part of a social space that can be characterized anthropologically, which has a significant impact on the interactions, and conversely, people rely on a number of assumptions related to their practices to interact.

INDEX

Mots-clés : Madagascar, Tananarive, analyse de conversation, communauté de pratiques, communication médiatisée par ordinateur (CMO), cybercafé, linguistique, malgache (langue) Keywords : Madagascar, Antananarivo, Conversation Analysis, Chat, Community, Computer Mediated Communication (CMC), Cybercafé, Linguistics, Malagasy

\section{AUTEUR}

\section{MAUD VERDIER}

Laboratoire Praxiling (UMR 5267), Université Paul Valéry Montpellier 3, Montpellier. 